Alimentary Tract

\title{
Myogenic oxidative imbalance interferes with antral motility in obese subjects
}

\author{
Annunziata Scirocco ${ }^{a}$, Lucia Pallotta ${ }^{b}$, Marco Rengo $^{c}$, Antonia Ignazzi $^{a}$, \\ Marilia Carabotti $^{\mathrm{b}}$, Alessia Cicenia ${ }^{\mathrm{b}}$, Rosa Vona ${ }^{\mathrm{d}}$, Piero Chirletti ${ }^{\mathrm{e}}$, \\ Maria Antonietta Maselli ${ }^{a}$, Rossella Donghia ${ }^{a}$, Mariagrazia Coluzzi ${ }^{\mathrm{f}}$, Paola Matarrese ${ }^{\mathrm{d}}$, \\ Gianfranco Silecchia ${ }^{\mathrm{f}}$, Carola Severi ${ }^{\mathrm{b}, *}$
}

a Experimental Pharmacology Laboratory, Scientific Institute of Gastroenterology “S. de Bellis”, Castellana Grotte, BA, Italy

${ }^{\mathrm{b}}$ Department of Internal Medicine and Medical Specialties, University Sapienza, Rome, Italy

${ }^{\mathrm{c}}$ Department of Radiological Sciences, Oncology and Pathology, I.C.O.T., University Sapienza, Latina, Italy

d Center for Gender-Specific Medicine, Istituto Superiore di Sanità, Rome Italy

e Department of General Surgery Francesco Durante, University Sapienza, Rome, Italy

${ }^{\mathrm{f}}$ Department of Medical Surgical Sciences and Biotechnology, University Sapienza, Latina, Italy

\section{A R T I C L E I N F O}

\section{Article history:}

Received 3 November 2017

Received in revised form 3 March 2018

Accepted 6 March 2018

Available online 14 March 2018

\section{Keywords:}

Human gastric smooth muscle cells

Obesity

Oxidative stress

Vasoactive intestinal peptide

\begin{abstract}
A B S T R A C T
Background: Obesity is characterized by a systemic low-grade chronic inflammatory oxidative condition that affects vascular and cardiac smooth muscle relaxation. In human antrum, relaxation is mediated by vasoactive intestinal peptide (VIP) through cAMP and cGMP signaling pathways. A genome-wide association study has demonstrated an association between VIP and obesity.

Aim: To evaluate smooth muscle activity in human obese antrum, both in in vitro preparations as well as in vivo.

Methods: Antral muscle strips and cells were isolated from surgical gastric samples from obese and normal weight subjects. Muscle contraction and relaxation, myogenic oxidative stress and inflammatory status were analyzed in vitro. Distal antral motility was evaluated in vivo by magnetic resonance imaging.

Results: Obese antral muscle cells showed an oxidative-inflammatory imbalance with overexpression of NLRP3 inflammasome, increased IL-1 $\beta$ secretion and caspase1-activation, and reduced antioxidant capacity associated with a myogenic motor impairment of VIP-induced relaxation. The intracellular alterations were characterized by a decreased activation of the cAMP-signaling pathway and a decreased expression of eNOS. These in vitro alterations coincided with the hindering of antral motor activity observed in vivo. Apocynin treatment, counteracting oxidative stress, reverted alterations observed in obese antral muscle.

Conclusion: Antral myogenic activity of obese subjects can be impaired by alterations of signaling pathways induced by oxidative stress.
\end{abstract}

(c) 2018 Editrice Gastroenterologica Italiana S.r.l. Published by Elsevier Ltd. All rights reserved.

\section{Introduction}

Obesity is a condition closely associated with a systemic low-grade chronic inflammatory oxidative condition [1]. Inflammation occurs as a result of immune cell infiltration into the adipose tissue and increased production of pro-inflammatory cytokines intrinsically linked to oxidative stress and responsible of obesity-derived metabolic complications. Oxidative stress and inflammatory cytokines are also considered as potential factors

\footnotetext{
* Corresponding author.

E-mail address: carola.severi@uniroma1.it (C. Severi).
}

in the induction of vascular dysfunction linked to hypertension, atherosclerosis, heart failure and diabetes [2].

Tissue inflammation occurring in obesity is apparently due to the nucleotide-binding oligomerization domain-like receptor family pyrin domain containing 3 (NLRP3) that, once activated by ROS, induces the release of the pro inflammatory cytokine IL-1 $\beta$ [3]. Generation of ROS, by reducing nitric oxide (NO) bioavailability, significantly affects vascular and cardiac smooth muscle relaxation $[4,5]$. The decreased NO production in obesity has also been ascribed to a reduced endothelial Nitric Oxide Synthase (eNOS) protein expression [6]. Furthermore, oxidative stress and inflammatory cytokines interfere in the regulation of G-protein coupled receptors (GPCR) that are essential molecules activating critical 
intracellular signaling pathways such as the cAMP and the phosphatidylinositol signaling pathways [7]. In particular, human antral smooth muscle relaxation is mainly mediated by vasoactive intestinal peptide (VIP) through its interaction with two subtypes of GPCRs receptors, $\mathrm{VPAC}_{2}$ and a single-transmembrane natriuretic peptide receptor $C$ (NPR-C) [8]. $\mathrm{VPAC}_{2}$ activates adenylyl cyclase and Protein Kinase A intracellular pathways whereas the NPR-C receptor activates $\mathrm{Ca}^{2+} /$ calmodulin-dependent eNOS, leading to NO formation and activation of soluble guanylyl cyclase and Protein Kinase G [9]. VIP binding to its receptors leads to the activation of both cGMP- and, more importantly, cAMP-dependent signaling pathway, which is essential for human antrum relaxation [8]. Of note, VIP pathway has been found to be significantly associated to obesity by a genome-wide association study [10].

The key hypothesis of the present study is that obesity-related inflammatory/oxidative imbalance may interfere with the VIPpathway induced relaxation of human antrum. Hence, smooth muscle impairment in obese antrum was evaluated by using both in vitro preparations (strips and cells) as well as in vivo in obese subjects by using magnetic resonance imaging.

\section{Materials and methods}

\subsection{Subjects}

The 2 groups of subjects were studied consisting of 15 morbid obese subjects submitted to sleeve gastrectomy (OB) and 13 normal weight subjects submitted to gastrectomy for gastric cancer $(\mathrm{NW})$, used as control group. Obesity was defined as $\mathrm{BMI} \geq 30 \mathrm{~kg} / \mathrm{m}^{2}$. All subjects gave written informed consent and the study was approved by the Ethical Committee (University Sapienza, rif.2903date: $26 / 09 / 2013$ ).

\subsection{Tissue preparations}

Gastric antral muscle obtained from the 2 groups of subjects was used for in vitro muscle preparations. Smooth muscle cells (SMC) and strips were isolated separately from the muscle layer of surgical gastric samples of the terminal antrum [8] and primary cultures of human SMC were prepared as previously described [11]. Surgical specimen from gastrectomy for gastric cancer were removed at least $5-6 \mathrm{~cm}$ from the neoplastic area at the histologically diseasefree margin of antral resection in NW patients [8].

\subsection{Measurement of contractile and relaxant muscle response}

\subsubsection{Strips}

Tone was raised on muscle strips, after the equilibration period, by a fixed dose of carbachol $\left(10 \mu \mathrm{moll}^{-1}\right)$ that was repeated after $30 \mathrm{~min}$ and several washing steps. After, strips were exposed to increasing concentrations of carbachol $\left(0.1-1000 \mu \mathrm{moll}^{-1}\right.$, cumulatively) in order to obtain concentration-response curves. Spontaneous phasic contraction of gastric antral strips was expressed as frequency $\left(\mathrm{min}^{-1}\right)$ and amplitude of contraction $\left(\mathrm{mN} \mathrm{cm}^{-2}\right)$. The contraction to carbachol was expressed as $\mathrm{mN} \mathrm{cm}{ }^{-2}$

Relaxation was evaluated in response to cumulated increasing concentrations of VIP $\left(0.01-3 \mu \mathrm{moll}^{-1}\right)$ added at a stable contraction plateau induced by $\mathrm{KCl}\left(80 \mathrm{mmoll}^{-1}\right)$, causing a tonic contractile response of the strip that was stable for at least $20 \mathrm{~min}$. After the contraction plateau was reached, VIP was added to the organ bath performing only one concentration response curve on each strip. The relaxation to VIP was expressed as a percentage decrease in the maximal contractile response to $\mathrm{KCl}$. At the end of each experiment, strips were removed, blotted and weighted.

\subsubsection{Cells and treatments}

Contraction and relaxation studies were performed as previously described [8]. The length of 50 cells in sequential microscopic fields was measured by image-scanning micrometry both in the control state and upon addition of tested agents using a ProgRes ${ }^{\circledR}$ camera with CapturePro 2.6 application software (Jenoptik Laser Optik, Jena, Germany) installed on a phase-contrast microscope (Leica Microsystems, Wetzlar, Germany). Contraction was expressed as percentage decrease in cell length from control taken as 100 and relaxation as percentage inhibition of contraction.

2.3.2.1. Apocynin exposure. To evaluate the contribution of oxidative stress, OB gastric SMC were incubated for $24 \mathrm{~h}$ in the absence or presence of the NADPH oxidase inhibitor apocynin (APO) $(60 \mu \mathrm{M})$ [12] and thereafter relaxation, antioxidant capacity, IL-1 $\beta$ secretion and eNOS transcript were re-evaluated. SMC without APO were considered as control.

2.3.2.2. Hydrogen peroxide treatment. To induce mild oxidative stress, cells were treated with $30 \mu \mathrm{M} \mathrm{H}_{2} \mathrm{O}_{2}$ for $30 \mathrm{~min}$. After this time samples were prepared for contractile activity analyses.

\subsection{Total RNA extraction and $q P C R$ on SMC}

Total RNA was harvested from NW and OB SMC, using Trizol (Invitrogen Life Technologies, Carlsbad, CA) and single-stranded cDNA was synthesized according to the manufacturer's protocol (Invitrogen Life Technologies). mRNAs were quantified using TaqMan Gene Expression Assays (Applied Biosystems), with specific human primers: nlpr3(Hs00918082_m1), pycard (Hs00203118_m1), caspase-1(Hs00354836_m1), IL-1 $\beta$ (Hs00174097_m1), eNOS (Hs01574659_m1). Data were analyzed and expressed as relative quantification (RQ) values obtained by determining $\Delta \mathrm{Ct}$ values followed by determining $\Delta \Delta \mathrm{Ct}$ values and then RQ values via the equation $2^{-\Delta \Delta \mathrm{Ct}}$.

\subsection{Antioxidant capacity and cytokine secretion measurement}

NW and OB SMC antioxidant capacity was determined by evaluation of the combined non-enzymatic antioxidant capacity by TAC colorimetric assay Kit (Cell Biolabs Inc. San Diego, CA, USA), which measures the sample's capacity to convert $\mathrm{Cu}^{+2}$ to $\mathrm{Cu}^{+1}$, following manufacturer's instructions. Results obtained by TAC assay are converted to trolox equivalents. IL- $1 \beta$ levels were evaluated by using a specific ELISA kit according to the manufacturer's protocol (Cayman; R\&D) on the culture medium of an equal cells number.

\subsection{Redox parameters}

\subsubsection{ROS}

Cells isolated from NW and OB patients were incubated in Hank's balanced salt solution, pH 7.4, with dihydrorhodamine 123 (DHR123, Thermo Fisher) in polypropylene test tubes for $15 \mathrm{~min}$ at $37^{\circ} \mathrm{C}$ (final concentration $10 \mu \mathrm{M}$ ). DHR 123 dye freely diffuses into cells and is primarily oxidized by $\mathrm{H}_{2} \mathrm{O}_{2}$ producing green fluorescence.

\subsubsection{GSH}

Following the same protocol, GSH intracellular content was evaluated by using monochlorobimane (MBC, Thermo Fisher). Samples were washed twice in ice-cold PBS and immediately acquired by an LRS II cytometer (Becton and Dickinson, San Jose, CA, USA) equipped with a UVB laser. 
Table 1

Demographics and clinical data of obese patients.

\begin{tabular}{|c|c|c|c|c|c|c|c|}
\hline & \multirow[t]{2}{*}{ Female } & \multirow[t]{2}{*}{ Age (years) } & \multirow[t]{2}{*}{ BMI kg/m² } & \multirow[t]{2}{*}{ Upper GI symptoms } & \multirow[t]{2}{*}{ PPI } & \multicolumn{2}{|l|}{ Antral histology } \\
\hline & & & & & & Acute inflammation & Chronic inflammation \\
\hline Patient 1 & $\sqrt{ }$ & 37 & 40.2 & None & & 0 & $1+$ \\
\hline Patient 2 & $\sqrt{ }$ & 46 & 51.5 & None & & 0 & 0 \\
\hline Patient 3 & $\sqrt{ }$ & 52 & 43.3 & None & & 0 & $1+$ \\
\hline Patient 4 & & 31 & 55.5 & None & & $1+$ & $1+$ \\
\hline Patient 5 & $\sqrt{ }$ & 19 & 41.5 & Globus & & 0 & 0 \\
\hline Patient 6 & $\sqrt{ }$ & 41 & 38.1 & None & & 0 & $1+$ \\
\hline Patient 7 & $\sqrt{ }$ & 36 & 45.3 & None & & 0 & 0 \\
\hline Patient 8 & $\sqrt{ }$ & 59 & 42.0 & None & & 0 & 0 \\
\hline Patient 9 & $\sqrt{ }$ & 32 & 43.7 & None & & 0 & 0 \\
\hline Patient 10 & $\sqrt{ }$ & 24 & 42.9 & None & & 0 & 0 \\
\hline Patient 11 & $\sqrt{ }$ & 42 & 42.5 & None & & 0 & 0 \\
\hline Patient 12 & & 31 & 36.0 & None & & 0 & $1+$ \\
\hline Patient 13 & $\sqrt{ }$ & 47 & 45.1 & None & $\sqrt{ }$ & $1+$ & $1+$ \\
\hline Patient 14 & $\sqrt{ }$ & 31 & 39.8 & None & & 0 & $1+$ \\
\hline Patient 15 & $\sqrt{ }$ & 23 & 44.8 & None & & 0 & $1+$ \\
\hline
\end{tabular}

BMI: body mass index; PPI: proton pump inhibitors; Hp: Helicobacter pylori.

\subsection{Activation of caspase-1}

Analysis of caspase-1 activation was performed by using a specific colorimetric kit (BioVision, Milpitas, CA) following manufacturer's instructions. Samples were read with a spectrophotometer at $400-405 \mathrm{~nm}$.

\subsection{Evaluation of protein expression levels}

Cell were fixed with 4\% paraformaldehyde (Carlo Erba) and then permeabilized with $0.5 \%$ Triton X-100 (Sigma-Aldrich). After washings, cells were incubated with the polyclonal antibodies to NLP3 (Sigma) or to capsase-1 (Millipore) for $1 \mathrm{~h}$ at $4{ }^{\circ} \mathrm{C}$. After washings, cells were incubated with anti-rabbit AlexaFluor 488conjugated (Termo Fischer) for additional $45 \mathrm{~min}$ at $37^{\circ} \mathrm{C}$. Samples were acquired by an LRS II cytometer (Becton and Dickinson).

\subsection{Magnetic resonance imaging and clinical gastrointestinal evaluation in obese subjects}

Distal antral contractile motility was evaluated with cine magnetic resonance imaging (MRI) as previously described [13]. After an overnight fast, healthy NW subjects and OB subjects drank a 650$\mathrm{ml}$ standardized liquid meal (Nutridrink) over 10 min plus two egg yolks and one albumen and $150 \mathrm{ml}$ of water (525-kcal, 25\% fat, 25\% protein, and $50 \%$ carbohydrate). Images were acquired before (T0), immediately after the end of the meal assumption (T1) and every $20 \mathrm{~min}$ (T2-T5), for a total exam time of $100 \mathrm{~min}$. Each gastric motility scan lasted $60 \mathrm{~s}$ with 100 images acquired in free breathing. The distance between base points along the antral wall provided the width of ACWs whereas the average distance between base points of ACWs across the antrum provided the antral diameters [13].

In OB subjects, upper gastrointestinal (GI) symptoms were assessed through a standardized questionnaire and upper GI symptoms were analyzed either separately or altogether to classify subjects in gastro-esophageal reflux disease and dyspepsia syndrome, as defined by Criteria of Rome III [14,15].

\subsection{Statistical analysis}

Values are presented as means \pm SE of duplicate examinations of n experiments, $n$ referring to the number of subjects from whom the stomach specimens were obtained to identify differences between OB and NW. Statistical analysis was performed by parametric and non-parametric ANOVA test, corrected for multiple comparisons by the Bonferroni procedure, significance being set at $p<0.05$. Cor- relation analysis was performed by parametric Pearson's test. For analysis, MRI scans were evaluated on a dedicated software to study geometry of distal antral waves contractions (ACWs) and grouped as T0, corresponding to fasting, T1-3 to the early $1 \mathrm{~h}$ post-prandial period and T4-5 to the late post-prandial period.

\section{Results}

OB subjects were significantly younger in comparison to NW [median age (range): 36 years (19-59) vs 55 years (42-63) respectively, $\mathrm{p}=0.0285$ ] and female gender was significantly prevalent in OB compared to NW, fourteen $v$ s one, respectively ( $\mathrm{p}=0.0003)$. In $\mathrm{OB}$ and $\mathrm{NW}$, BMI [median $\mathrm{kg} / \mathrm{m}^{2}$ (range)] was 42.90 (36.0-55.5) and 21.6 (19.8-24.0), respectively.

None of the $\mathrm{OB}$ subjects presented comorbidities (diabetes mellitus; dyslipidemia; cardiovascular disease; hypertension) or assumed drugs (nitrates, calcium antagonist, $\beta$ blockers, prokinetics) with potential effects on GI motility. At pre-surgical antral histology (Table 1), half of the obese patients showed mild mucosal chronic inflammation and only two of them a low-grade active inflammation, without Helicobacter pylori infection.

\subsection{Inflammasome components and oxidative stress in obese human antral smooth muscle}

The mRNA expression of nlrp3 and its co-molecules, pycard and caspase- 1 were overexpressed in human OB gastric SMC compared to NW (Fig. 1A). An increased expression of IL-1 $\beta$ mRNA was also observed in OB SMC. In addition, it was observed the secretion of IL-1 $\beta$ in culture medium of OB SMC $\left(11.07 \pm 1.98 \mathrm{pg} \mathrm{ml}^{-1}\right)$, which was not detectable in SMC from NW subjects. As far as protein expression level was concerned, a significant increase of NLRP3 was observed in human OB gastric SMC compared to NW (Fig. 1B). By contrast, the increase of caspase-1 protein level observed in $\mathrm{OB}$ was not statistically significant, but a significant up-regulation of the active form of caspase- 1 was found in OB subjects, indicating an activation of inflammasome (Fig. 1B).

Further, in comparison to NW subjects, a reduced antioxidant capacity was detected in gastric SMC from OB subjects $(-55.22 \pm 0.84 \%$ vs NW). Accordingly, in comparison with NW subjects, a significant increase of ROS production $(+61.34 \pm 7.12 \%$, with a parallel reduction of GSH intracellular levels $(-49.4 \pm 5.04 \%)$ were detected, indicating a condition of oxidative stress in SMC isolated from $\mathrm{OB}$. 
A

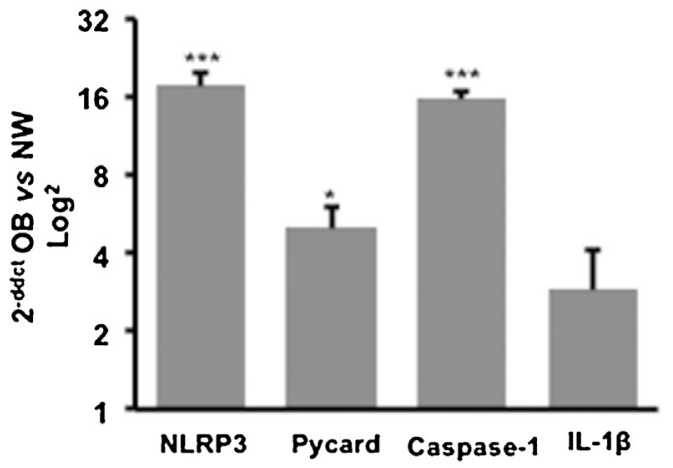

B

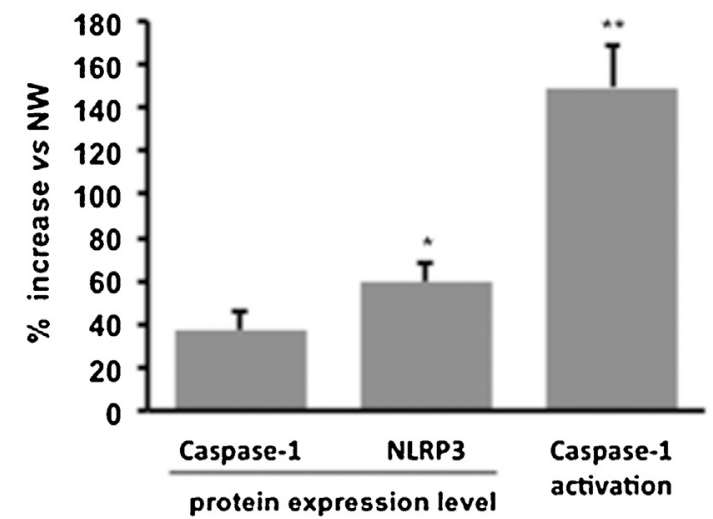

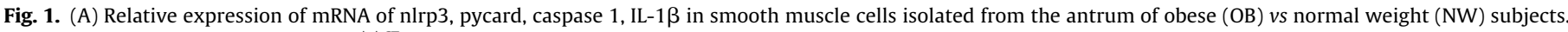

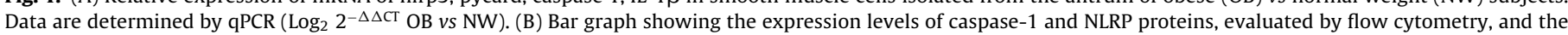

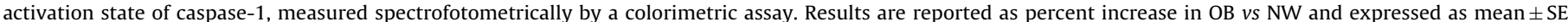

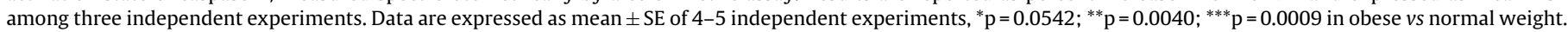

\subsection{Contractile activity of human $O B$ gastric antral smooth muscle}

Muscle strips from NW and OB subjects displayed an intrinsic myogenic activity with phasic contractions of $3.96 \pm 0.88$ and $5.96 \pm 1.23 \mathrm{mN} \mathrm{cm}^{-2}$ with an amplitude of $2.11 \pm 0.45$ and $0.64 \pm 0.15 \mathrm{~min}^{-1}$, respectively $(\mathrm{p}=0.0134)$. In these samples, the maximum response to carbachol was significantly different $(p=0.0001)$. In fact, it was reached at a concentration of $1000 \mu \mathrm{mol} \mathrm{l}^{-1}$, with $1022.0 \pm 59.70 \mathrm{mN} \mathrm{cm}^{-2}$, in NW subjects, and at a concentration of $100 \mu \mathrm{moll}^{-1}$, with $107.9 \pm 39.15 \mathrm{mN} \mathrm{cm}^{-2}$, in OB subjects. No differences were instead observed between SMC from NW and $\mathrm{OB}$ antrum either in resting cell lengths (77.83 \pm 5.13 and $74.90 \pm 2.92 \mu \mathrm{m}$, respectively) or in maximal contractile responses $(25.33 \pm 1.78$ and $26.27 \pm 1.54 \%$, respectively).

\subsection{Relaxant activity of human gastric antral smooth muscle}

VIP caused a dose-dependent relaxation of both NW and OB muscle strips that was however significantly reduced in samples from OB. Representative traces of VIP-induced inhibition of contractile activity in muscle strips in NW and OB subjects are shown in Fig. 2A and B. In OB strips, maximal relaxation induced by VIP was inhibited by $57.15 \%$ in comparison with NW (Fig. 3A) and almost completely absent in SMC from OB subjects (Fig. 3B). The impaired relaxant muscle response to VIP in SMC was not correlated to blood glycemia ( $r=-0.29,95 \% \mathrm{CI}$ : -0.890 to 0.686 ; $\mathrm{p}=0.587)$ or total cholesterol levels ( $r=0.2457,95 \% \mathrm{CI}$ : -0.706 to $0.8815 ; \mathrm{p}=0.638)$.

To further identify the effects of oxidative stress on VIP-induced relaxation in SMC, chemically-induced oxidative stress was investigated on SMC of NW subjects after exposure to $30 \mu \mathrm{M}$ hydrogen peroxide for $30 \mathrm{~min}$, an experimental condition able to induce caspase-1 activation in human gastric SMC (data not shown). After exposure to hydrogen peroxide, VIP-induced relaxation was inhibited by $69.79 \pm 9.7 \%$ in comparison with control buffer.

\subsection{VIP-associated signaling transduction pathways}

In order to better analyze the impairment of VIP-induced antrum relaxation in $\mathrm{OB}$, the effects of direct activators of cyclases and of the 2nd messengers of the two VIP-related transduction pathways in human antrum [8] were tested, separately, in the absence of VIP and the expression of eNOS, a key molecule in cGMPtransduction signaling, was analyzed by qPCR.
Table 2

Magnitude of diameters variations and width of antral contraction waves during fasting (T0), $1 \mathrm{~h}$ (T1-T3) and $2 \mathrm{~h}$ (T4-T5) postprandially. Data are mean $\pm \mathrm{SE}$, ${ }^{*} \mathrm{p}=0.0001$ obese $(\mathrm{OB})$ vs normal weight (NW) subjects.

\begin{tabular}{llllll}
\hline & \multicolumn{2}{l}{ Diameters variations $(\mathrm{cm})$} & & \multicolumn{2}{l}{ Antral contraction waves $(\mathrm{cm})$} \\
\cline { 2 - 3 } \cline { 5 - 6 } & OB & NW & & OB & NW \\
\hline T0 & $0.238 \pm 0.013^{*}$ & $0.302 \pm 0.013$ & & $0.988 \pm 0.020^{*}$ & $1.216 \pm 0.021$ \\
T1-3 & $0.246 \pm 0.012^{*}$ & $0.341 \pm 0.015$ & & $1.057 \pm 0.021^{*}$ & $1.394 \pm 0.021$ \\
T4-5 & $0.248 \pm 0.013^{*}$ & $0.307 \pm 0.012$ & & $0.933 \pm 0.020^{*}$ & $1.261 \pm 0.021$ \\
\hline
\end{tabular}

OB SMC underwent an impairment of the cAMP-dependent transduction pathway, both at cyclase and second messenger level. Relaxation induced by the adenylate cyclase activator FSK was significantly inhibited $(-26.79 \pm 7.53 \%)$ in OB vs NW SMC. By contrast, it was significantly increased by cAMP $(+41.22 \pm 4.35 \%$ in OB vs NW, Fig. 3C). Instead, no alterations were observed in the cGMP-dependent transduction pathway relaxation induced by the guanylate cyclase activator SNP, being similar in OB and NW cells. Of note, cGMP caused a higher relaxation in $\mathrm{OB}$ in comparison to NW cells (Fig. 3D). The quantitative real-time PCR also showed a decreased expression of eNOS mRNA in human gastric OB SMC compared to NW $\left(2^{-\Delta \Delta \mathrm{Ct}}\right.$ OB $\left.v s \mathrm{NW}: 0.11 \pm 0.05\right)$.

\subsection{Antral contractions and clinical gastrointestinal evaluation in obese subjects}

In OB subjects, the motility scans showed a hindered antral motor activity both during fasting and postprandial periods in term of magnitude of antral diameter variations and ACWs (Table 2). The reduced muscle dynamism was reflected by significantly reduction of magnitude of antral diameter variations and ACWs width in OB in comparison with NW subjects, both during fasting (T0) and post-prandial (T1-3 and T4-5) periods. Negative correlations have been found between antral ACWs width and ROS $(r=-0.961,95 \% \mathrm{CI}$ : -0.996 to $0.679 ; \mathrm{p}=0.0023)$ or caspase 1 -activation $(\mathrm{r}=-0.901$, $95 \% \mathrm{CI}$ : -0.989 to $0.332 ; \mathrm{p}=0.0142$ ), whereas a positive correlation between ACWs width and GSH content $(r=-0.939,95 \% \mathrm{CI}$ : $0.538-0.993 ; p=0.0054$ ) was observed. Furthermore, the postprandial increase in ACWs width, observed in the early first hour post-prandial period (T1-3) with respect to the corresponding $\mathrm{T} 0$, was significantly less in OB $(7.20 \pm 0.60 \%)$ than in NW subjects $(14.73 \pm 0.38 \%)$.

This reduced antral motility observed in OB subjects was not associated to defined clinical presentations in that the Rome II 


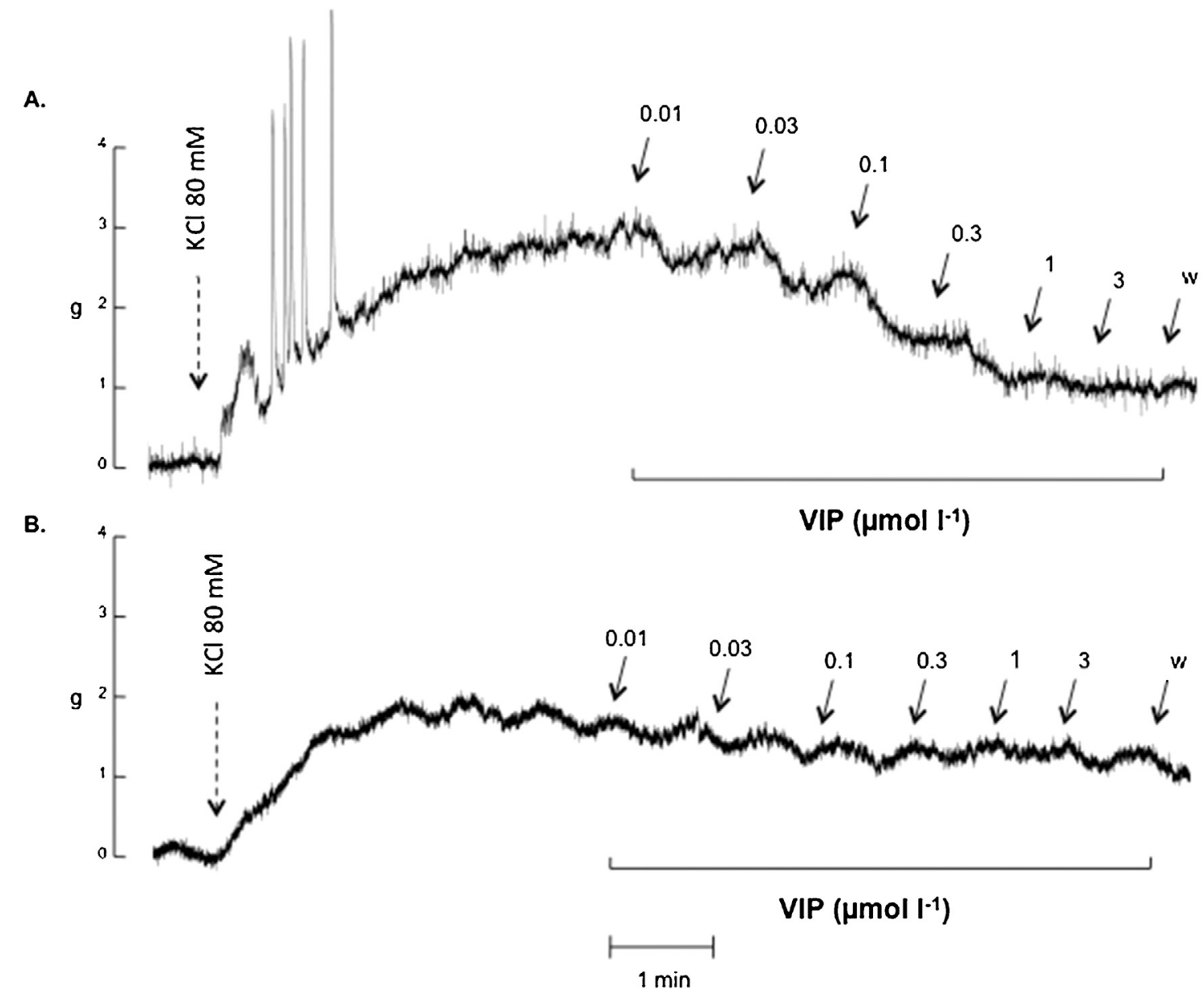

Fig. 2. Representative traces of VIP-induced inhibition of human antral muscle strips contractile activity in normal weight (A) and obese (B) subjects.

Modular Questionnaire for functional disorders showed that $90.9 \%$ (10/11) of OB subjects did not complain upper gastrointestinal symptoms. In NW subjects, given the presence of gastric cancer, clinical symptoms have not been considered.

\subsection{Oxidative stress contribution to the impaired relaxation}

To establish the potential role of oxidative stress in gastric myogenic impairment occurring in samples from OB subjects, apocynin (APO), a specific inhibitor of NADPH oxidase, was used for in vitro experiments, as previously tested in SMC preparations [12] (Fig. 4). A $24 \mathrm{~h}$-incubation of OB SMC with APO $(60 \mu \mathrm{M})$ was able to restore relaxant responses to VIP, FSK and cAMP to values similar to those observed in NW SMC. APO administration also increased antioxidant capacity in OB SMC, significantly decreased their secretion of inflammatory cytokine IL-1 $\beta$, and "normalized" eNOS expression (Fig. 4).

\section{Discussion}

This study provides the first direct evidence that antral smooth muscle relaxation is impaired in obese subjects and that this is apparently due to a muscular oxidative imbalance that mainly affects the cAMP-signaling pathway and the expression of eNOS, two key components of VIP-induced relaxation [9]. These alterations that were observed in both muscle strips and SMC probably lead to a hindered antral muscle dynamics in vivo with a reduction in width of antral contraction waves and diameter variations. The key role played by oxidative stress in the observed muscle alter- ations was supported by the ability of apocynin to restore in vitro myogenic relaxation and by the positive correlation found between ACWs widths and the altered inflammatory/oxidative balance in OB subjects.

Several studies have reported that a pro-oxidant inflammatory environment was present in obesity and correlations have been established between free radicals-mediated oxidative stress and body mass index or high fat diet [16]. In vascular muscle of OB subjects, the pro-oxidative imbalance leads to the activation of NLRP3 inflammasome, and the release of active IL- $1 \beta$ yielding to a proinflammatory microenvironment [1]. The present study highlights that a similar condition could also occur in antral muscle from $\mathrm{OB}$ subjects and that this was not related to the low grade of mucosal inflammation observed in some OB subjects, since similar muscle alterations were present in all patients regardless of their mucosal features.

In vascular muscle of OB subjects, the oxidative-inflammatory status was reported to affect human antral motor apparatus in different ways. It has been observed that myogenic relaxation was impaired and this was mainly due to the dysfunction of the cAMPsignaling pathway. As a consequence, the responses to FSK, an activator of the adenylate cyclase, and to the 2nd messenger, were significantly reduced. An impairment of the cAMP-signaling following oxidative stress has already been reported in human endothelial cells [17] and in human airways SMC [18] as a consequence either of an increased activity of phosphodiesterases that inactivate cAMP, or of an interference of IL- $1 \beta$ on cAMP generation. The lack of eNOS expression, previously observed in obese human [5] and rodent [19] muscle tissues, could also contribute to determine the impair- 

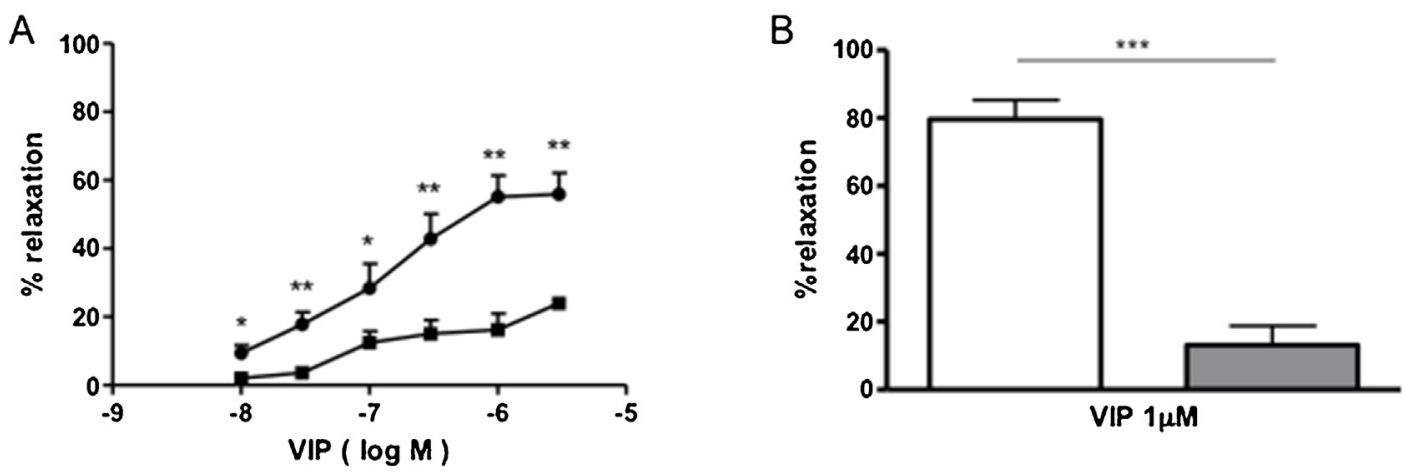

C
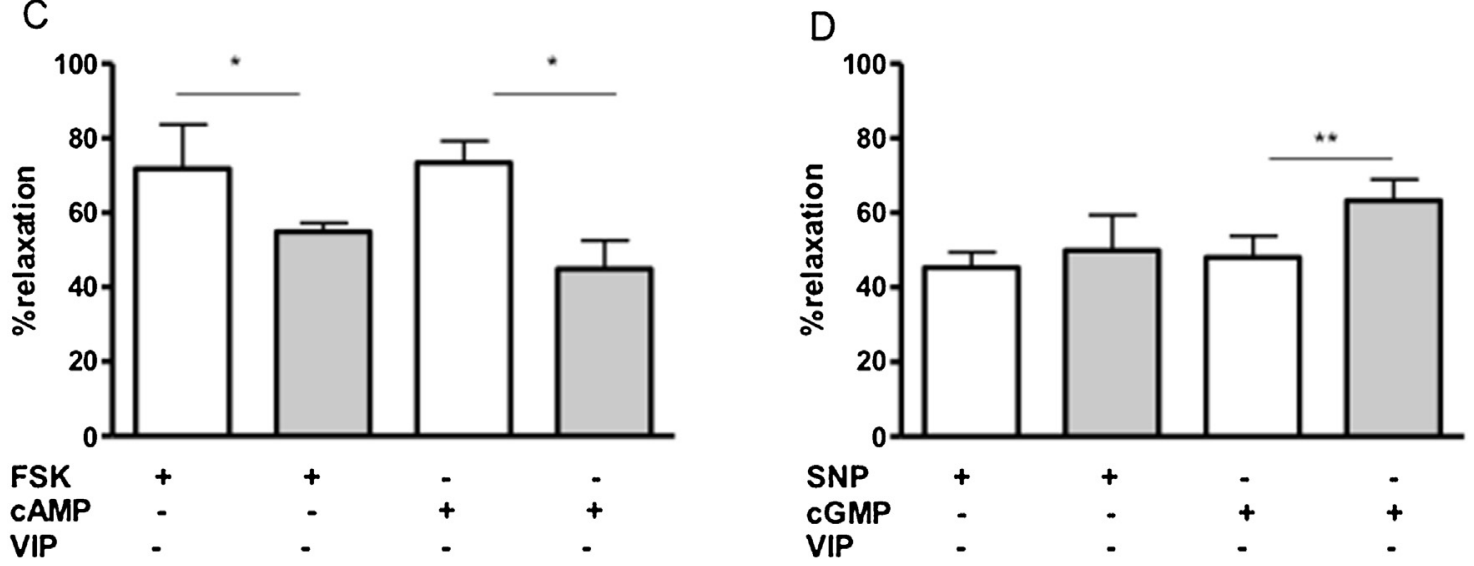

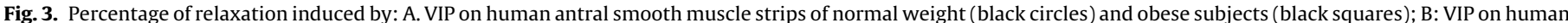

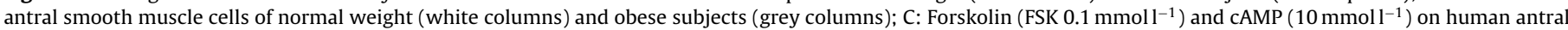

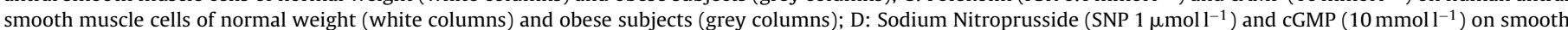

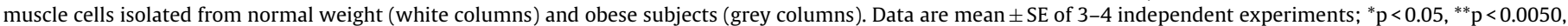
${ }^{* * *} \mathrm{p}<0.0001$ obese $v s$ normal weight.

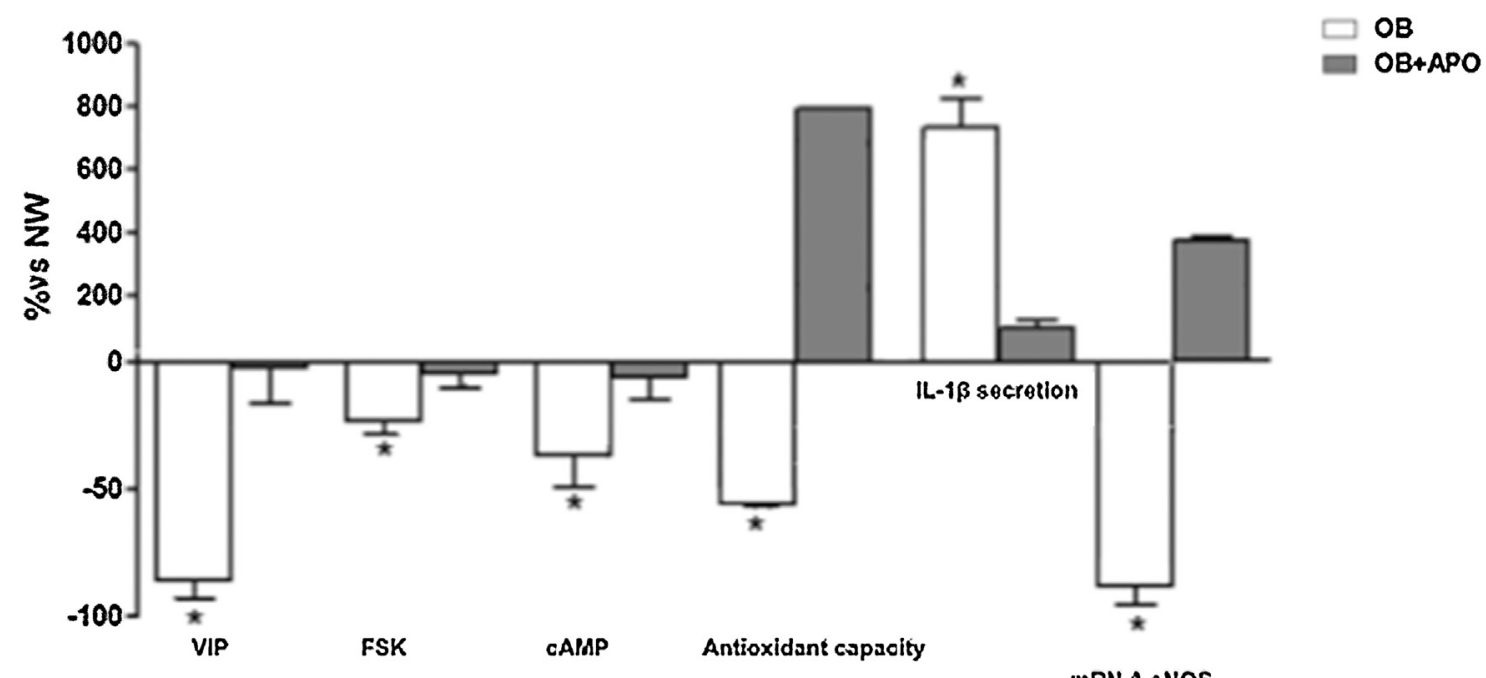

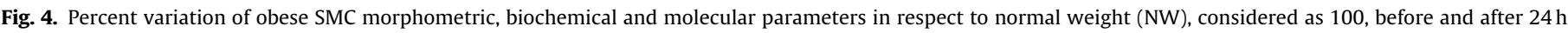

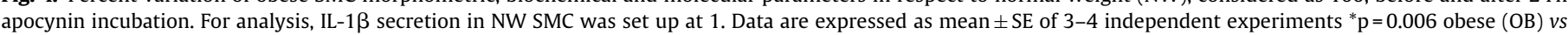
obese + apocynin $(\mathrm{OB}+\mathrm{APO})$.

ment of muscle relaxation observed in obese antrum. In fact, it has been reported that inflammatory cytokines decrease the stability of eNOS mRNA [20], and an excess of adipose tissue, by blunting NO production, causes a decrease in skeletal muscle activity [21] and microvascular dysfunctions [1]. Further, an increase of ROS production reduced the amount of bioactive nitric oxide, converting it to toxic peroxynitrite, which could uncouple eNOS from signaling [22].

The present study shows that in antral SMC from OB subjects the impairment of eNOS expression impeded VIP-induced activation of the cGMP-signaling pathway, that resulted functionally unchanged since a similar relaxation occurred in OB and NW SMC in response 
to SNP, an activator of the guanylate cyclase. The increased efficacy of the 2nd messenger cGMP, we observed in antral OB SMC, has previously been reported in cardiac smooth muscle cells, in which oxidative stress activated the cGMP-dependent kinase [18].

The key role of oxidative stress in the impairment of human $\mathrm{OB}$ antral muscle functionality was also confirmed by the fact that the inhibition of NADPH oxidase activity, the main enzyme involved in production of reactive oxygen species, by apocynin reverted either the impairment of antioxidant cell capacity or inflammatory cytokine IL- $1 \beta$ secretion or eNOS down regulation in vitro, restoring a physiologic relaxation response. The effectiveness of apocynin in restoring VIP-induced relaxation could therefore be ascribed to its ability to normalize both the cAMP signaling and eNOS expression. The great increase in the cellular antioxidant capacity after apocynin treatment is in accordance with its ability to up-regulate the synthesis of glutathione, a potent intracellular antioxidant [23].

A further consequence of the oxidative-inflammatory status was the altered contraction observed in human smooth muscle strips, but not SMC. This seems to suggest that the alterations in $\mathrm{OB}$ antral neuromuscular apparatus also involved the myenteric plexus. Accordingly, IL-1 $\beta$ has been reported to affect Ach-induced intestinal contraction through a neurally-mediated mechanism on the myenteric plexus that not involve directly smooth muscle [24].

The impaired relaxation of SMC observed in vitro coincided with a hindered antral motility with reduction in width of antral contraction waves and diameters variations observed in vivo during fasting and post-prandial periods. Although a significant correlation was found between ACWs and muscle oxidative imbalance during fasting, the direct involvement of oxidative stress in ACWs should be confirmed on a larger number of samples before being considered biologically significant. This antimotility condition is further supported by the intrinsic myogenic activity of muscle strips in which the increase in the force resulted associated to a $70 \%$ decrease in the amplitude of spontaneous contractions in respect to NW [25]. A similar condition appears to play a significant role in coronary microvascular dysfunction observed in obesity [26]. The hindered antral muscle dynamics did not appear to be associated to upper gastrointestinal symptoms, in particular dyspepsia, considering that all the obese subjects we analyzed did not present relevant clinical symptoms. Thus, the contribution of antral motility in the pathophysiology of dyspepsia remains secondary despite the prevalent role of gastric fundic relaxation [27]. In fact, no differences in proximal stomach function have been reported between $\mathrm{OB}$ and NW subjects [28]. In addition, the absence of impairment of the cGMP-dependent pathway in OB muscle reported in the present study further strengthens that the relaxation of proximal stomach is likely not affected [8]. However, the absence of symptoms in OB subjects may also be related to the impaired visceral sensation, ascribing to a dysfunction of the autonomic nervous system. According with this, in OB subjects both manometric and endoscopic esophageal alterations have been reported to occur predominantly in the absence of GI symptoms [29,30].

Our study has limitations in terms of the heterogeneity of the subjected analyzed. In fact, OB and NW subjects presented differences in terms of age and gender, with a greater prevalence of the female gender in the obesity group and a higher prevalence of older males in NW subjects undergoing to surgery for gastric cancer. The higher prevalence of women in the OB group is in accordance with the global gender disparity that shows, in developed countries, a prevalence of $\mathrm{OB}$ women in comparison to men [31]. In addition, it has also been observed that $\mathrm{OB}$ men underuse bariatric surgery. As far as age is concerned, OB subjects candidate to bariatric surgery are often younger than subjects with gastric cancer whose prevalence increases with age.

In conclusion, this study demonstrates for the first time that myogenic alterations occurring in antral muscle in OB subjects could mainly be ascribed to ongoing oxidative stress that impairs relaxation. Similar alterations might occur in other visceral muscles and further studies are required to elucidate the functional significance of the observed motor alterations. Further, a novel functional link between VIP and obesity has been established, supporting the genome analyses [10], that could be important in the understanding the possible role of this pleiotropic neurotransmitter with multiple physiological activities in determining wide biological alterations.

\section{Conflict of interests}

\section{None declared.}

\section{Funding}

This article was supported by a grant from University Sapienza, Roma Italy to CS (grant number 000324_13Severi Sapienza).

\section{Disclosure}

None declared.

\section{Acknowledgment}

The authors thank Prof. Enrico Corazziari, alumnus of the University Sapienza, for the critical revision of the manuscript.

\section{References}

[1] Savini I, Catani MV, Evangelista D, Gasperi V, Avigliano L. Obesity-associated oxidative stress: strategies finalized to improve redox state. Int J Mol Sci 2013; $14: 10497-538$

[2] De Boer MP, Meijer RI, Wijnstok NJ, Jonk AM, Houben AJ, Stehouwer CD, et al. Microvascular dysfunction: a potential mechanism in the pathogenesis of obesity-associated insulin resistance and hypertension. Microcirculation 2012:19:5-18.

[3] Vandanmagsar B, Youm YH, Ravussin A, Galgani JE, Stadler K, Mynatt RL, et al. The NLRP3 inflammasome instigates obesity-induced inflammation and insulin resistance. Nat Med 2011;17:179-88.

[4] Wadley AJ, Veldhuijzen van Zanten JJ, Aldred S. The interactions of oxidative stress and inflammation with vascular dysfunction in ageing: the vascular health triad. Age (Dordr) 2013;35:705-18.

[5] Wu J, Xia S, Kalionis B, Wan W, Sun T. The role of oxidative stress and inflammation in cardiovascular aging. Biomed Res Int 2014;2014:615312.

[6] Georgescu A, Popov D, Constantin A, Nemecz M, Alexandru N, Cochior D, et al. Dysfunction of human subcutaneous fat arterioles in obesity alone or obesity associated with type 2 diabetes. Clin Sci 2011;120:463-72.

[7] Pascual RM, Billington CK, Hall IP, Panettieri Jr RA, Fish JE, Peters SP, et al. Mechanisms of cytokine effects on $\mathrm{G}$ protein-coupled receptor-mediated signaling in airway smooth muscle. Am J Physiol Lung Cell Mol Physiol 2001;281:1425-35.

[8] Severi C, Tattoli I, Corleto VD, Maselli MA, Trisolini P, Delle Fave G. Vasoactive intestinal peptide receptor subtypes and signalling pathways involved in relaxation of human stomach. Neurogastroenterol Motil 2006;8:1009-18.

[9] Murthy KS. Signaling for contraction and relaxation in smooth muscle of the gut. Annu Rev Physiol 2006;68:345-74.

[10] Liu YJ, Guo YF, Zhang LS, Pei YF, Yu N, Yu P, et al. Biological pathway-based genome-wide association analysis identified the vasoactive intestinal peptide (VIP) pathway important for obesity. Obesity 2010;18:2339-46.

[11] Tattoli I, Corleto VD, Taffuri M, Campanini N, Rindi G, Caprilli R, et al. Optimisation of isolation of richly pure and homogeneous primary human colonic smooth muscle cells. Dig Liver Dis 2004;36:735-43.

[12] Scirocco A, Matarrese P, Petitta C, Cicenia A, Ascione B, Mannironi C, et al. Exposure of toll-like receptors 4 to bacterial lipopolysaccharide (LPS) impairs human colonic smooth muscle cell function. J Cell Physiol 2010;223:442-50.

[13] Kwiatek MA, Steingoetter A, Pal A, Menne D, Brasseur JG, Hebbard GS, et al. Quantification of distal antral contractile motility in healthy human stomach with magnetic resonance imaging. J Magn Reson Imaging 2006;24:1101-9.

[14] Drossman DA, Talley NJ, Whitehead WE, Corazziari E. The Rome II Modular Questionnaire: investigator and respondent forms. In: Rome II the functional gastrointestinal disorders. McLean-Degnon Associates; 2000.

[15] Tack J, Talley NJ, Camilleri M, Holtmann G, Hu P, Malagelada JR, et al. Functional gastroduodenal disorders. Gastroenterology 2006;130:1466-79.

[16] Kesh SB, Sarkar D, Manna K. High-fat diet-induced oxidative stress and its impact on metabolic syndrome: a review. Asian J Pharm Clin Res 2016;9:47-52.

[17] Muzaffar S, Jeremy JY, Angelini GD, Shukla N. NADPH oxidase 4 mediates upregulation of type 4 phosphodiesterases in human endothelial cells. Cell Physiol 2012;227:1941-50. 
[18] Pang L, Holland E, Knox AJ. Role of cyclo-oxygenase-2 induction in interleukin$1 \beta$ induced attenuation of cultured human airway smooth muscle cell cyclic AMP generation in response to isoprenaline. Br J Pharmacol 1998;125:1320-8.

[19] Valerio A, Cardile A, Cozzi V, Bracale R, Tedesco L, Pisconti A, et al. Tnf-alpha downregulates enos expression and mitochondrial biogenesis in fat and muscle of obese rodents. J Clin Invest 2006;116:2791-8.

[20] Lai PE, Mohamed F, Monge JC, Stewart DJ. Downregulation of eNOS mRNA expression by TNFalpha: identification and functional characterization of RNAprotein interactions in the 3'UTR. Cardiovasc Res 2003;59:160-8.

[21] Hickner RC, Kemeny G, Stallings HW, Manning SM, McIver KL. Relationship between body composition and skeletal muscle eNOS. Int J Obes 2006;30:308-12.

[22] Förstermann U. Nitric oxide and oxidative stress in vascular disease. Pflugers Arch 2010;459:923-39.

[23] Lapperre TS, Jimenez LA, Antonicelli F, Drost EM, Hiemstra PS, Stolk J, et al. Apocynin increases glutathione synthesis and activates AP-1 in alveolar epithelial cells. FEBS Lett 1999;443:235-9.

[24] Aubé AC, Blottière HM, Scarpignato C, Cherbut C, Rozé C, Galmiche JP. Inhibition of acetylcholine induced intestinal motility by interleukin 1 beta in the rat. Gut 1996;39:470-4
[25] Deachapunya C, Thongsaard W, Poonyachoti S. Barakol suppresses norepinephrine-induced inhibition of spontaneous longitudinal smooth muscle contractions in isolated rat small intestine. J Ethnopharmacol 2005:101:227-32.

[26] Knudson JD, Dincer UD, Bratz IN, Sturek M, Dick GM, Tune JD. Mechanisms of coronary dysfunction in obesity and insulin resistance. Microcirculation 2007; 14:317-38.

[27] Talley N, Ford AC. Functional dyspepsia. N Engl J Med 2015;373:1853-63.

[28] Iovino P, Angrisani L, Galloro G, Consalvo D, Tremolaterra F, Pascariello A, et al. Proximal stomach function in obesity with normal or abnormal oesophageal acid exposure. Neurogastroenterol Motil 2006;18:425-32.

[29] Koppman JS, Poggi L, Szomstein S, Ukleja A, Botoman A, Rosenthal R. Esophageal motility disorders in the morbidly obese population. Surg Endosc 2007;21:761-4.

[30] Carabotti M, Avallone M, Cereatti F, Paganini A, Greco F, Scirocco A, et al. Usefulness of upper gastrointestinal symptoms as a driver to prescribe gastroscopy in obese patients candidate to bariatric surgery. A prospective study. Obes Surg 2016;26:1075-80.

[31] Kanter R, Caballero B. Global gender disparities in obesity: a review. Adv Nutr 2012;3:491-8 